\title{
In vivo efficacy of sulphadoxine-pyrimethamine for the treatment of asymptomatic parasitaemia in pregnant women in Machinga District, Malawi
}

Julie Gutman ${ }^{1 *}$, Dyson Mwandama ${ }^{2}$, Ryan E Wiegand ${ }^{1}$, Joseph Abdallah³ ${ }^{3}$ Nnaemeka C Iriemenam¹, Ya Ping Shi ${ }^{1}$, Don P Mathanga ${ }^{2,4}$ and Jacek Skarbinski ${ }^{1}$

\begin{abstract}
Background: The effectiveness of sulphadoxine-pyrimethamine (SP) intermittent preventive treatment of malaria in pregnancy (IPTp) might be compromised by high prevalence of resistance-associated Plasmodium falciparum dihydrofolate reductase (dhfr) and dihydropteroate synthase (dhps) mutations. As a proxy for IPTp-SP effectiveness, the in vivo efficacy of SP to clear parasitaemia and prevent reinfection in asymptomatic parasitaemic pregnant women in an area with high SP resistance prevalence was assessed.

Methods: Pregnant women 16-26 weeks' gestation with asymptomatic parasitaemia presenting for antenatal care were given IPTp-SP and followed for 42 days. The primary outcome was polymerase chain reaction (PCR) uncorrected 42-day survival rate; the per cent of patients without recrudescence or reinfection by day 42 . PCR was used to distinguish recrudescence from reinfection. DNA was sequenced to detect resistance-associated dhfr and dhps mutations.

Results: Of 245 pregnant women included in the intention-to-treat analysis, 93.9\% cleared their parasitaemia by day 7. The day 42 PCR-uncorrected survival rate was 58.1\% (95\% confidence interval (CI) 51.5-65.7) and day 42 PCR-corrected survival was $68.7 \%$ (Cl 61.4-76.0). Recrudescence was more common among primi- than among multigravid women; recrudescence rate $33.3 \%$ (Cl 25.1-42.4\%) versus $21.4 \%$ (Cl 15.0-29.0\%) (log rank test p-value 0.006). The quintuple mutant was present in nearly all samples $(95 \%)$, while $2 \%$ were sextuple mutants with an additional mutation at dhps A581G.
\end{abstract}

Conclusions: SP efficacy for acute malaria treatment has been compromised by resistance, but SP retains partial activity among pregnant women with asymptomatic parasitaemia, and thus might be useful for IPTp. Nonetheless, research on non-SP IPTp regimens should continue.

Trial registration: ClinicalTrials.gov NCT01120145.

Keywords: Malaria, Pregnancy, Intermittent-preventive treatment, Sulphadoxine-pyrimethamine, in vivo, Malawi

\section{Background}

Malaria in pregnancy is associated with severe maternal anaemia, placental malaria, low birth weight (LBW), maternal mortality, and infant morbidity and mortality, particularly among primigravid women [1]. Intermittent preventive treatment with sulphadoxine-pyrimethamine (IPTp-SP) reduces the adverse consequences of malaria

\footnotetext{
* Correspondence: jgutman@cdc.gov

${ }^{1}$ Malaria Branch, Division of Parasitic Diseases \& Malaria, Centers for Disease Control and Prevention, Atlanta, GA, USA

Full list of author information is available at the end of the article
}

among pregnant women, including third-trimester maternal anaemia, placental parasitaemia, and the prevalence of LBW infants [2-4]. When this study was conducted, the policy in Malawi was to give SP at the first antenatal care (ANC) visit after quickening (about 20 weeks) and again during the third trimester, with a total of two doses during pregnancy; this has since been updated in line with revised WHO recommendations to provide IPTp-SP at each scheduled ANC visit starting early second trimester with a minimum of three doses during pregnancy [5]. 
Resistance to SP is mediated by the sequential accumulation of single nucleotide polymorphisms (SNPs) that encode amino acid substitutions in the Plasmodium falciparum dihydropteroate synthase (dhps) and dihydrofolate reductase (dhfr) genes; the quintuple mutant haplotype (dhfr mutations N51I, C59R, S108N, and dhps mutations A437G and $\mathrm{K} 540 \mathrm{E}$ ), which is associated with failure of SP in in vivo studies in children, has been fixed in Malawi since 2005 [6,7]. Due to the high level of SP resistance, SP has not been recommended for treatment of symptomatic malaria in Malawi since 2007. Nonetheless, studies suggest that SP remains effective as IPTp even when it is no longer effective as a treatment in symptomatic children $[2,8]$. However, studies from both Tanzania and Malawi suggest IPTp-SP effectiveness could be compromised by the presence of additional mutations conferring increased resistance to SP [9-11]; in particular, the presence of an additional mutation at dhps A581G (the sextuple mutant) seems to significantly compromise the effectiveness of IPTp-SP [10,12,13].

As resistance to SP increases, it is important to monitor the efficacy of IPTp-SP to determine when this intervention no longer provides any benefit. However, there is no standardized methodology for monitoring. In vivo efficacy in children does not correlate well with the degree of protection afforded to pregnant women [2], because pregnant women have partial immunity, and thus other methods to monitor IPTp-SP effectiveness are needed. As a proxy for SP effectiveness, a 42-day in vivo efficacy study was conducted among asymptomatic pregnant women to assess the efficacy of SP for clearing existing parasitaemia and preventing new infection. As pregnancy specific anti-parasitic immunity increases with each pregnancy, efficacy was assessed separately among primigravid and multigravid women.

\section{Methods \\ Study site}

The study was conducted in the antenatal clinic of Machinga District Hospital, Malawi from March 2010 to January 2011. Residents are mainly of the Yao ethnic group, who earn their living through subsistence farming, fishing and small businesses. Machinga District Hospital is the primary hospital for a catchment area including 369,614 people, with approximately 5,712 deliveries per year. In 2011 , there were 57,999 clinically diagnosed malaria cases, of which 29,626 (51\%) were aged $\geq$ five years [12]. Malaria transmission in the district is stable throughout the year with a peak in the rainy season, from December to March. Parasite prevalence was $42.3 \%$ in children aged < five years in the Southern Region in the most recent malaria indicator survey [12]. The vast majority of malaria infections are caused by Plasmodium falciparum. Resistance to SP is widespread; the quintuple mutation is fixed, although at the time of this study the dhfr I164L mutation had not been detected and the dhps A581G mutation was detected at extremely low frequency $(<1 \%)[6]$.

\section{Sample size}

Parasitologic failure rate among pregnant women treated with SP was 11\% in 2004, double the failure rate in 1996 (5\%) [14]. Allowing for the possibility that failure rates had increased, a sample size of 300 women was determined to be sufficient to estimate the proportion of failures with a $95 \%$ confidence interval (CI) width of 0.10 , assuming a $20 \%$ failure rate, and allowing for approximately $20 \%$ loss to follow-up [15].

\section{Enrolment and follow-up}

Pregnant women of all parities presenting to Machinga District Hospital for ANC visits were eligible for inclusion if they: 1) were between 16 and 26 weeks' gestation (based on last menstrual period (LMP) or quickening); 2) were eligible to take SP on the day of enrolment; 3) had malaria parasitaemia of any density; 4) had an axillary temperature below $37.5^{\circ} \mathrm{C}$; 5) had a viable foetus documented by detection of a foetal heartbeat or foetal movement; 6) were HIV-negative; 7) had not received any anti-malarials or antibiotics with anti-malarial activity in the previous month; 8) had no history of SP hypersensitivity; and, 9) provided informed consent for participation. At enrolment, a finger-prick blood specimen was collected for malaria rapid diagnostic test (RDT) (Paracheck-Pf Device (Orchid Biomedical Systems, India)), malaria microscopy, haemoglobin measurement (HemoCue 201, HemoCue AB, Sweden), and dried blood spot (DBS) for polymerase chain reaction (PCR); three tablets of SP (a total of $1,500 \mathrm{mg}$ sulphadoxine and $75 \mathrm{mg}$ of pyrimethamine) was administered by directly observed therapy. Quality-assured SP was procured from Durbin Plc, Middlesex, UK.

Following enrolment, patients were assessed weekly until day 42 or until they developed parasitaemia. At each visit, women were assessed for fever (axillary temperature $\geq 37.5^{\circ} \mathrm{C}$ ) or histoy of fever and medication use, and a finger-prick blood specimen was collected for thick smear, haemoglobin measurement, and DBS for PCR to distinguish reinfection from recrudescence. Thick blood smears were prepared with Field's stain and the number of parasites per 200 white blood cells (WBC) counted. Blood smears were considered negative if no parasites were found after counting 1000 WBC. Blood smears were read by two independent microscopists with a third read in the case of discordant results (discrepancy between positivity/negativity or greater than $50 \%$ difference in parasite counts). Parasite density was calculated as the average of the closest two readings, assuming an average WBC count of $8,000 / \mathrm{mm}^{3}$. Participants were 
instructed to return to the clinic immediately if they felt sick. Women with positive blood smears at any time on or after day 4 were treated with artemether-lumefantrine per national guidelines.

\section{Molecular analyses}

Resistance to SP was measured by direct sequencing of DNA extracted from DBSs collected from the day of enrolment. The DNA samples were sequenced at $d h f r$ codons 51, 59, 108, 164 and dhps codons 436, 437, 540, 581, 613 using a standard protocol [16]. The quintuple mutant was defined as an isolate containing dhfr mutations N51I, C59R and S108N, and dhps mutations A437G and K540E [17]. The presence of an additional mutation at dhps A581G defined the sextuple mutant.

In order to differentiate recrudescence from reinfection, DNA samples extracted from paired DBS samples from enrolment (day 0) and day of failure were assessed using WHO-recommended PCR procedures for testing polymorphisms at the genes encoding merozoite surface protein 1 (msp-1) and msp-2 [18].

\section{Statistical analysis}

As IPTp-SP is designed to both treat prevalent parasitaemia as well as prevent incident parasitaemia, the main outcome of interest was clearance of parasites without recrudescence or reinfection in the 42 days following SP administration (survival), which differs from the standard WHO in vivo, in which the main outcome of interest is clearance of parasites without recrudescence (i.e. treat prevalent infection only) [15]. Data analysis was done in SAS version 9.3 (Cary, NC, USA) and R version 3.0.1 (Vienna, Austria). Survival curves were calculated using the Kaplan-Meier method; in absence of failure, women were censored on the last day for which data were available [19]. The log-rank test was used to assess for significant differences between curves. Multiple imputation (with 10 imputed datasets) was used to assess the sensitivity of results when incorporating cases where PCR testing for recrudescence versus reinfection was unsuccessful [20]. Cox proportional hazards regression was used to test for predictors of failure [21]. The final, multivariable model was chosen to reflect predictors which improve the fit of the model based on Schwarz's Bayesian Information Criterion [22]. For all analyses, a p-value of $<0.05$ considered significant. Plots were generated with the ggplot2 package [23].

\section{Ethics}

The study was approved by the ethical review boards of the University of Malawi College of Medicine (Blantyre, Malawi) and the Centers for Disease Control and Prevention (Atlanta, GA, USA). Written informed consent was obtained from all participating women prior to enrolment. The study was registered at ClinicalTrials. gov (NCT01120145).

\section{Results}

A total of 2,566 women were screened; P. falciparum antigenaemia was detected by RDT in 759 (29.6\%). Of the 301 women who met all inclusion criteria and consented to enrolment, 299 were enrolled; 58 women were lost prior to the first follow-up, and were excluded from the analysis. A total of 245 pregnant women were included in the analyses (Figure 1). Of these, 114 (46.5\%) were primigravid (G1) and 131 (53.5\%) were multigravid (G2+) (Table 1). The mean age was 21.7 years (range 14-42) overall, 18.8 years (range 14-38) among primigravid women and 24.3 years (range 16-42) among multigravid women; $54.3 \%$ were less than or equal to 20 years old. In all, $32.2 \%$ reported having used a bed net on the night prior to enrolment; $84.8 \%$ of these were insecticide-treated nets (ITNs). Bed net use was higher among multigravid than among primigravid women ( 41.2 versus $21.9 \%, \mathrm{p}=0.001$ ). The mean gestational age at enrolment was 21 weeks (IQR 18-24); multigravid women were enrolled on average 1.5 weeks later in gestation than primigravid women $(\mathrm{p}=0.002)$.

\section{Recrudescence and reinfection}

During the course of the study, 63 women (25.7\%) remained aparasitaemic through day 42 while 87 women developed parasitaemia (35.5\%) of whom 51 (58\%) had recrudescent infection, 17 (20\%) were reinfected, and in 19 (22\%) PCR was unavailable (13 samples could not be amplified and six were unavailable). Reinfection occurred at a median of 15 days (interquartile range $14-28$ days). A total of 95 women were censored prior to reaching a valid study end point; 43 women were lost to follow-up, and another 52 women were withdrawn due to protocol violation (46 were given IPTp-SP at day 35 and six received anti-malarial treatment without evidence of malaria or another antibiotic with anti-malarial activity).

Overall, $93.9 \%$ of women cleared their infection by day 7; the PCR-uncorrected 28-day survival rate was $67.5 \%$ (Table 2). The PCR-uncorrected 42-day survival rate was $58.1 \%$; the survival rate was $45.8 \%$ in primigravid women and $68.0 \%$ in multigravid women. Over 42 days, there was a significant association between survival and gravidity (log-rank Chi-sq $=11.5, \mathrm{df}=1, \mathrm{p}=0.0007$ ) (Figure 2). PCR-corrected survival, including multiple imputations for missing PCR results, was $76.4 \%$ (CI 70.2-82.6) at day 28 and $68.7 \%$ (CI 61.4-76.0) at day 42. Recrudescence was more common among primi- than among multigravid women, with a recrudescence rate of $44.0 \%$ (CI 31.6$56.3 \%$ ) and $23.6 \%$ (CI 14.9-32.3\%), respectively (log-rank Chi-sq $=9.3, \mathrm{df}=1, \mathrm{p}=0.002$ ) by day 42 . 


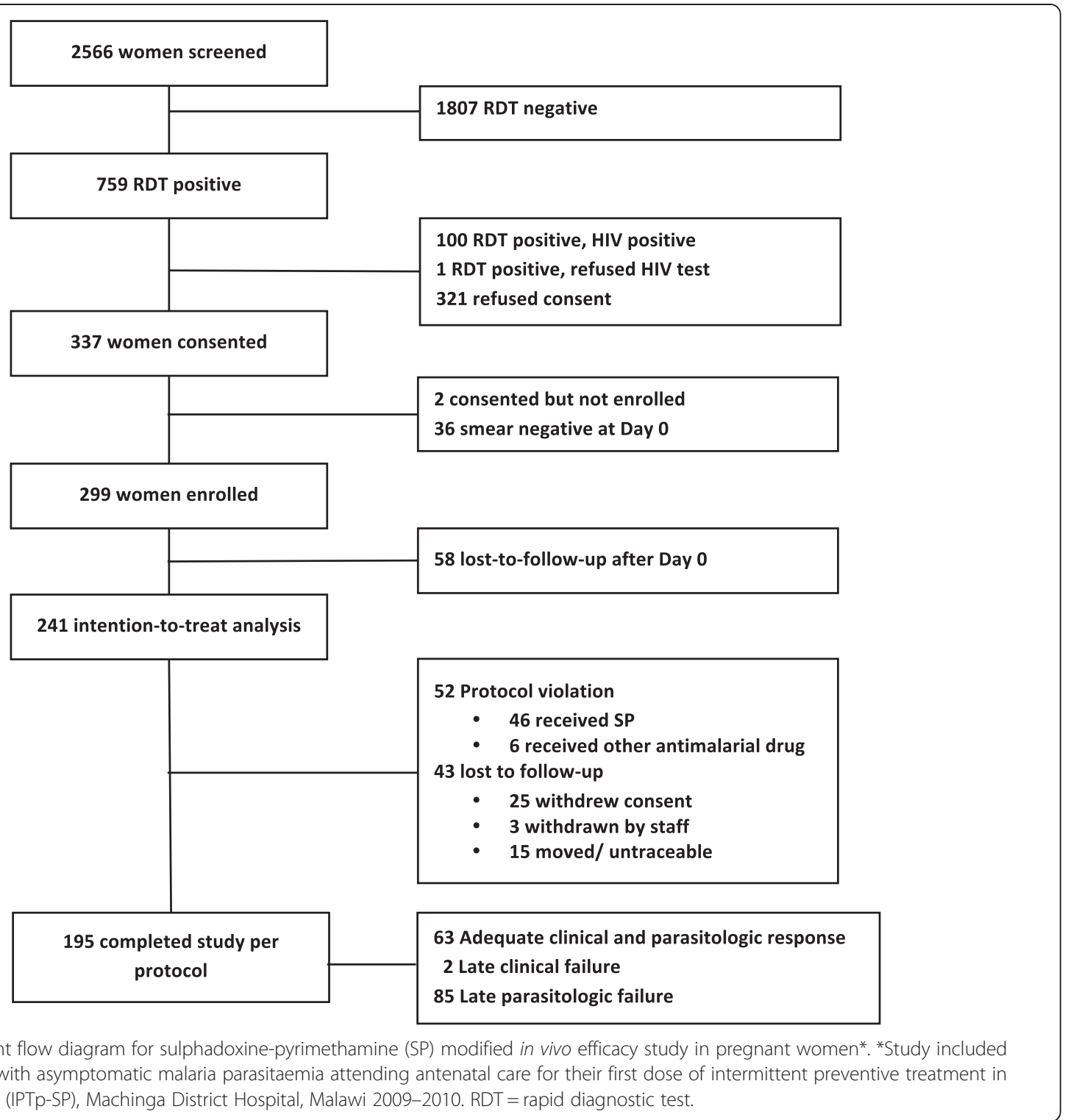

Both higher gravidity and bed net use were shown to be protective against developing recrudescence or reinfection in univariate modelling, however, in the multivariate model, only gravidity remained significant (Table 3 ). When examining the risk of recrudescence only, as expected, bed net use was no longer a significant factor, and only gravidity remained significant in both univariate and multivariate analyses.

\section{Haematologic response}

Mean haemoglobin increased by $0.3 \mathrm{mg} / \mathrm{dL}$ (95\% CI 0.1 $0.4, p=0.0001)$ from day 0 until the last day of followup. The change was greater among primigravid women (0.4 $\mathrm{mg} / \mathrm{dL}$ (95\% CI 0.2-0.7) than among multigravid women $(0.1 \mathrm{mg} / \mathrm{dL}(95 \% \mathrm{CI}-0.05-0.3), \mathrm{p}=0.02)$. A significant interaction was found between day of follow-up and gravid class $(\mathrm{F}(6,243)=3.86, \mathrm{p}=0.0011)$ indicating the change in haemoglobin levels during the study was not the same in each group. Figure 3 shows the raw means with $95 \%$ confidence intervals across the two groups.

\section{Molecular markers of SP resistance}

Of the 245 day 0 samples, a variable number could be amplified at each codon. Where samples were amplified, there was a very high frequency of mutation $(\geq 97 \%)$ at each of the following codons: dhfr-51, dhfr-59, dhfr-108, dhps-437, and dhps-540 (Table 4). Of 182 samples that were successfully genotyped at all five codons, 95\% carried the quintuple mutant. Four samples (2\%) (one from a primigravid woman and three from multigravid women), all of which carried the quintuple mutation, were mutated at dhps-581 indicating the presence of sextuple mutants. 
Table 1 Baseline characteristics of asymptomatic parasitaemic pregnant women enrolled in a modified in vivo efficacy study of sulphadoxine-pyrimethamine (SP), stratified by gravidity

\begin{tabular}{|c|c|c|c|c|}
\hline & Total $(\mathrm{N}=245)$ & Primigravid $(\mathrm{N}=114)$ & Multigravid $(\mathrm{N}=131)$ & p-value \\
\hline Age, mean (SD) & $21.7(5.0)$ & $18.8(2.9)$ & $24.3(5.0)$ & $<0.0001$ \\
\hline Age $\leq 20$ years & $54.3 \%$ & $87.7 \%$ & $25.2 \%$ & $<0.0001$ \\
\hline \multicolumn{5}{|l|}{ Bed net use } \\
\hline Used a bed net last night & $32.2 \%$ & $21.9 \%$ & $41.2 \%$ & 0.001 \\
\hline Used an insecticide-treated bed net last night & $27.3 \%$ & $14.9 \%$ & $38.2 \%$ & $<0.0001$ \\
\hline Maternal weight $(\mathrm{kg})$, mean (SD) & $55.8(7.1)$ & $55.4(6.9)$ & $56.1(7.3)$ & 0.40 \\
\hline Education (years), mean (SD) & $6.2(3.0)$ & $6.9(2.9)$ & $5.6(3.0)$ & $<0.001$ \\
\hline Perceived below average wealth status & $78.0 \%$ & $81.6 \%$ & $74.6 \%$ & 0.19 \\
\hline Gestational age at enrolment (weeks), mean (SD) & $21.0(3.9)$ & $20.2(3.6)$ & $21.8(4.0)$ & 0.002 \\
\hline Haemoglobin at enrolment, mean (SD) & $9.8(1.3)$ & $9.2(1.1)$ & $10.4(1.3)$ & $<0.0001$ \\
\hline Per cent of women with anaemia $(\mathrm{Hb}<11 \mathrm{~g} / \mathrm{dL}$ ) at enrolment & $81.2 \%$ & $96.5 \%$ & $67.9 \%$ & $<0.0001$ \\
\hline Per cent of women with gametocytaemia & $0.4 \%$ & $0.00 \%$ & $0.8 \%$ & - \\
\hline Parasite density (parasites/ml), geometric mean (range) & $273(32-12,835)$ & $372(48-12,835)$ & $209(32-8,955)$ & $<0.0001$ \\
\hline
\end{tabular}

$\mathrm{SD}=$ standard deviation, $\mathrm{Hb}=$ haemoglobin.

One of these patients had adequate clinical and parasitologic response (ACPR), two had a treatment failure (one on day 7 and the other on day 8), and one was censored on day 35 due to a protocol violation. The presence of the sextuple mutant was detected in three additional samples from the day of failure (one from a primigravid woman and two from multigravid women). Unfortunately, the day-of-failure samples from the two patients who had sextuple mutants detected at baseline and subsequently failed therapy could not be amplified at codon dhps-581.

\section{Discussion}

Overall, 58.1\% of pregnant women who presented with asymptomatic parasitaemia and received SP on day 0 remained aparasitaemic for 42 days, in an area with a high prevalence of SP resistance (95\% carried the quintuple mutant, $2 \%$ carried the sextuple mutant at baseline). Parasite-free survival rates were higher among multigravid (68\%) than among primigravid (46\%) women. Although 28-day survival was $67.5 \%$, well below the $90 \%$ WHO threshold for changing the recommendation for first-line treatment of uncomplicated malaria [24], this study suggests that SP retains some efficacy among pregnant women, and that monthly dosing of IPTp-SP, as recommended by WHO [25], likely continues to provide benefit. This is supported by a recent study conducted at the same site assessing the effect of IPTp-SP on birth outcomes, which found a dose-dependent protective effect of IPTp-

Table 2 Polymerase chain reaction survival rates by week among asymptomatic parasitaemic women treated with sulphadoxine-pyrimethamine at day 0 , stratified by gravidity

\begin{tabular}{llll}
\hline Uncorrected & & & \\
\hline Day of follow-up & Primigravid survival rate $\mathbf{( 9 5 \% ~ C l )}$ & Multigravid survival rate $\mathbf{( 9 5 \% ~ C l )}$ & Overall survival rate (95\% Cl) \\
\hline $\mathbf{7}$ & $92.1 \%(87.3-97.2)$ & $95.4 \%(91.9-99.1)$ & $93.9 \%(90.9-96.9)$ \\
$\mathbf{1 4}$ & $76.5 \%(68.8-85.0)$ & $88.3 \%(82.9-94.0)$ & $82.9 \%(78.2-87.9)$ \\
$\mathbf{2 8}$ & $58.5 \%(49.6-69.1)$ & $75.0 \%(67.6-83.2)$ & $67.5 \%(61.6-74.0)$ \\
$\mathbf{3 5}$ & $48.6 \%(39.3-60.0)$ & $71.2 \%(63.4-79.9)$ & $61.1 \%(54.8-68.1)$ \\
$\mathbf{4 2}$ & $45.8 \%(36.1-58.2)$ & $68.0 \%(59.5-77.6)$ & $58.1 \%(51.5-65.7)$ \\
\hline Corrected & & & Overall survival rate $\mathbf{( 9 5 \% ~ C l )}$ \\
\hline Day of follow-up & Primigravid survival rate $\mathbf{( 9 5 \% ~ C l )}$ & Multigravid survival rate $\mathbf{( 9 5 \% ~ C l )}$ & $96.5 \%(93.6,99.4)$ \\
\hline $\mathbf{7}$ & $95.9 \%(91.0,100.0)$ & $97.1 \%(93.9,100.0)$ & $88.2 \%(83.6,92.8)$ \\
$\mathbf{1 4}$ & $84.2 \%(76.5,91.9)$ & $91.4 \%(86.2,96.7)$ & $76.4 \%(70.2,82.6)$ \\
$\mathbf{2 8}$ & $68.3 \%(58.3,78.3)$ & $82.8 \%(75.5,90.1)$ & $70.2 \%(63.5,77.0)$ \\
$\mathbf{3 5}$ & $59.3 \%(48.1,70.5)$ & $78.6 \%(70.6,86.6)$ & $68.7 \%(61.4,76.0)$ \\
$\mathbf{4 2}$ & $56.0 \%(43.7,68.3)$ & $76.4 \%(67.7,85.0)$ & \\
\hline
\end{tabular}

$\mathrm{Cl}$ : confidence interval. 


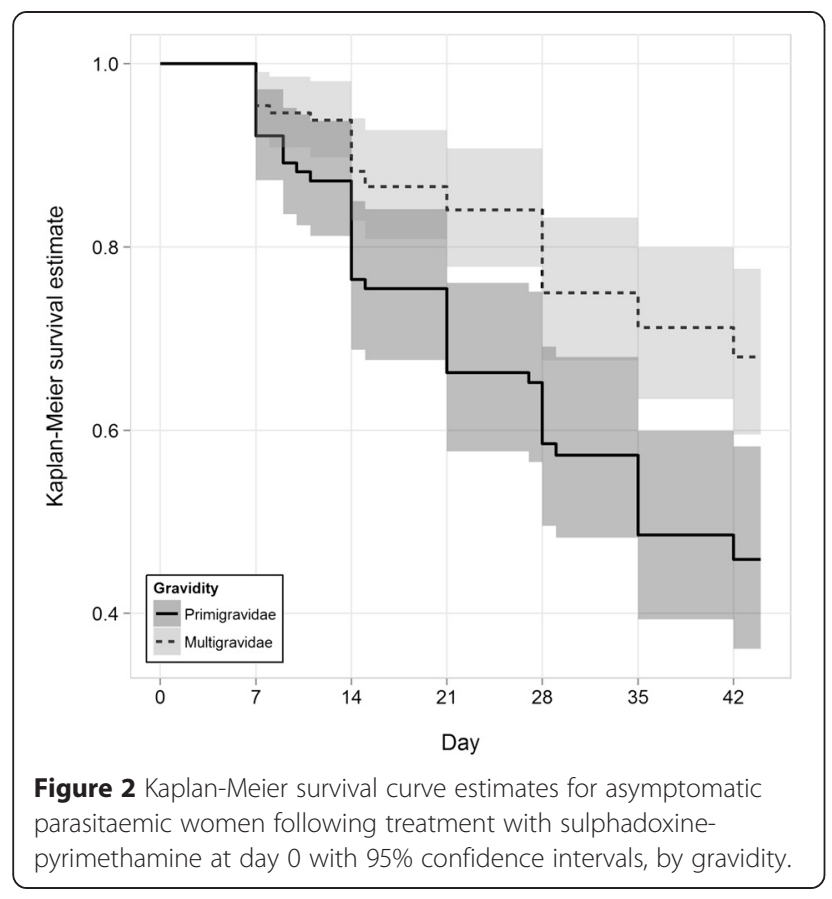

SP among primigravid women on a composite birth outcome of LBW, preterm delivery, and small for gestational age (SGA) [26]. Similarly, a recent meta-analysis found a greater benefit of IPTp-SP when three or more doses were given during pregnancy compared to two, irrespective of the prevalence of the quintuple mutation [8].

Although the presence of the quintuple mutant predicts failure of SP treatment among children aged $<$ five years with acute malaria [17], IPTp-SP remains effective even where the quintuple mutant is highly prevalent [8].
In this study, due to the extremely high prevalence of the quintuple mutant, it was not possible to assess whether this haplotype was associated with a higher rate of failure; despite the high prevalence of the quintuple mutant, IPTp-SP continues to improve birth outcomes in this area [26]. In Tororo, Uganda, where $>90 \%$ of parasites carry the quintuple mutant, Arinatwe et al. also found a significant reduction in a composite adverse outcome measure composed of placental malaria, LBW, maternal parasitaemia, or maternal anaemia [27]. A study assessing SP in vivo efficacy among asymptomatic, parasitaemic pregnant women in Zambia, where the quintuple mutant was present in $61 \%$ of samples at baseline, found no association of the quintuple mutant with treatment failure [28]. In that study setting, IPTp-SP was associated with a dose-dependent reduction in LBW, with a greater impact among primi- and secundigravid compared to multigravid women [29].

Although IPTp-SP remains effective even in the presence of the quintuple mutant, several studies suggest that the presence of the sextuple mutant is associated with decreased effectiveness of IPTp-SP, as evidenced by either increased parasite densities, increased placental inflammation, or decreased birth weight among children born to women infected with parasites carrying the sextuple mutant $[10,12,13]$. In an area with extremely high levels of resistance in Muheza, Tanzania, where the quintuple mutant is fixed and the mean fraction of resistant alleles at dhps 581 was 0.31, IPTp-SP was not associated with a statistically significant benefit at the population level [11]. The lack of efficacy may reflect the effect of the high prevalence of the sextuple mutant or alternatively may have been due to insufficient power in

Table 3 Factors associated with treatment failure among asymptomatic parasitaemic women treated with sulphadoxine-pyrimethamine at day 0

\begin{tabular}{|c|c|c|c|c|c|c|c|c|}
\hline & \multicolumn{4}{|c|}{ Univariable } & \multicolumn{4}{|c|}{ Multivariable } \\
\hline & \multirow[b]{2}{*}{ HR } & \multicolumn{2}{|l|}{$95 \% \mathrm{Cl}$} & \multirow[b]{2}{*}{ p-value } & \multirow[b]{2}{*}{ HR } & \multicolumn{2}{|l|}{$95 \% \mathrm{Cl}$} & \multirow[b]{2}{*}{ p-value } \\
\hline & & Lower & Upper & & & Lower & Upper & \\
\hline \multicolumn{9}{|l|}{ Treatment failure, uncorrected by PCR } \\
\hline ITN use last night (reference: no ITN use last night) & 0.54 & 0.32 & 0.92 & 0.02 & 0.67 & 0.38 & 1.16 & 0.16 \\
\hline Age: comparing $>20$ years to $\leq 20$ years & 0.68 & 0.44 & 1.06 & 0.09 & & & & \\
\hline Schooling years (continuous) & 1.01 & 0.94 & 1.09 & 0.72 & & & & \\
\hline Log parasite density (continuous) & 0.96 & 0.79 & 1.17 & 0.70 & & & & \\
\hline Gravidity: comparing multigravid to primigravid & 0.48 & 0.31 & 0.74 & $<0.001$ & 0.53 & 0.34 & 0.83 & 0.006 \\
\hline \multicolumn{9}{|l|}{ Treatment failure, corrected by PCR } \\
\hline ITN use last night (reference: no ITN use last night) & 0.54 & 0.27 & 1.09 & 0.09 & 0.73 & 0.35 & 1.52 & 0.40 \\
\hline Age: comparing $>20$ years to $\leq 20$ years & 0.62 & 0.35 & 1.10 & 0.10 & & & & \\
\hline Schooling years (continuous) & 1.05 & 0.95 & 1.15 & 0.35 & & & & \\
\hline Log parasite density (continuous) & 0.97 & 0.75 & 1.25 & 0.79 & & & & \\
\hline Gravidity: comparing multigravid to primigravid & 0.41 & 0.23 & 0.73 & 0.002 & 0.45 & 0.25 & 0.82 & 0.009 \\
\hline
\end{tabular}

$\mathrm{HR}=$ hazard ratio, $\mathrm{Cl}=$ confidence interval, $\mathrm{ITN}=$ insecticide-treated bed net, $\mathrm{PCR}=$ polymerase chain reaction. 


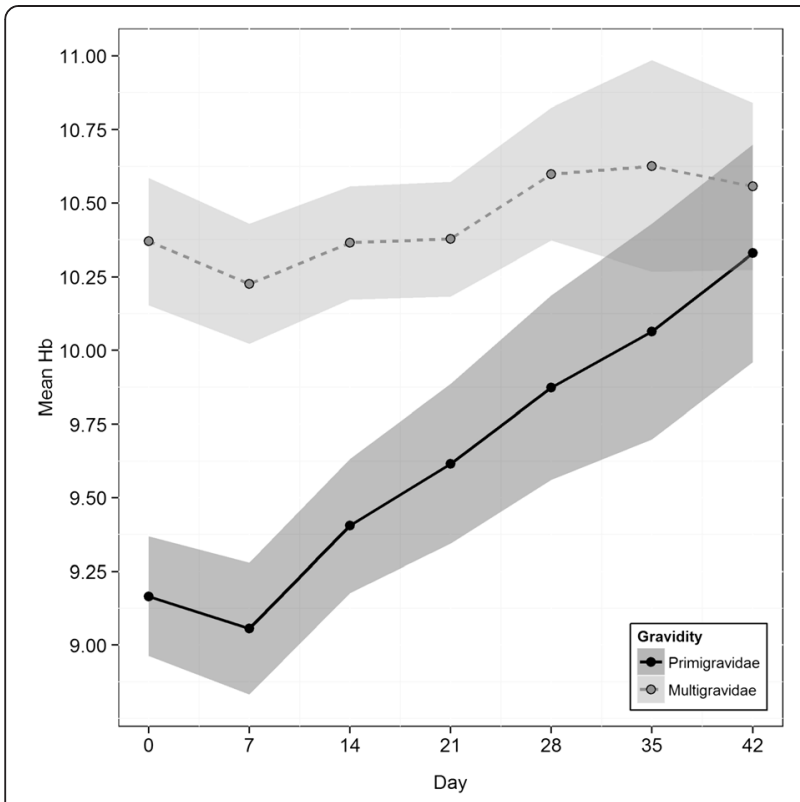

Figure 3 Mean haemoglobin levels with 95\% confidence intervals broken down by gravidity.

the study, as the point estimates for the effect on LBW favour IPTp. As the prevelance of SP resistance continues to increase, additional studies are needed to assess the true effect of the sextuple mutant on IPTp-SP efficacy, and the prevalence at which a population level effect is seen.

The protective effect of IPTp-SP is thought to be a result of both the ability to clear existing infections as well as to prevent new infections. Some have argued that the prophylactic effect is of primary importance [30].

\begin{tabular}{|c|c|c|}
\hline Mutation & Mutant (n)/Total (N) & Per cent mutant \\
\hline dhfr N51I & $209 / 212$ & $99 \%$ \\
\hline dhfr C59R & $210 / 214$ & $98 \%$ \\
\hline dhfr S108N & $211 / 214$ & $99 \%$ \\
\hline dhfr I164L & $2 / 211$ & $1 \%$ \\
\hline dhps A437G & $219 / 225$ & $97 \%$ \\
\hline dhps K540E & $201 / 204$ & $99 \%$ \\
\hline dhps A581G & $4 / 204$ & $2 \%$ \\
\hline dhps A613T/S & $2 / 180$ & $1 \%$ \\
\hline Double* & 187/193 & $97 \%$ \\
\hline Triple* & $196 / 202$ & $97 \%$ \\
\hline Quintuple* & $172 / 182$ & $95 \%$ \\
\hline
\end{tabular}

*Double mutant $=$ Pfdhps mutations A437G and G540E; Triple mutant $=$ Pfdhfr mutations N511, C59R and S108N; Quintuple mutant = Pfdhfr mutations N511, C59R, S108N and Pfdhps mutations A437G and K540E.
However, a recent study from Malawi found that IPTp-SP was more effective in clearing existing sub-microscopic infections than preventing new sub-microscopic infections [31]. Moreover, intermittent screening with a RDT and treatment only of parasitaemic women has been shown to have similar benefits to IPTp-SP in preventing LBW and severe maternal anaemia, even when using a treatment with a short-acting drug such as artesunate-amodiaquine or artemether-lumefantrine [32]. (Tagbor et al., A noninferiority, individually randomized trial of intermittent screening and treatment versus intermittent preventive treatment in the control of malaria in pregnancy, submitted). This suggests that the treatment effect might be more important than the prophylactic effect. In this study, only $6.1 \%$ of women had persistent infection on day 7 , indicating that the vast majority of infections were suppressed, despite the high prevalence of parasite resistance to SP. SP was effective at clearing infections likely due to the fact that the infections were of low density; however immunity also played a role given the differential rate of cure between primi- and multigravid women.

Across the reported in vivo efficacy studies among pregnant women, there appears to be a correlation between failure rate and the prevalence of SP resistance mutations (Desai $\mathrm{M}$, et al.: Impact of sulfadoxinepyrimethamine resistance on effectiveness of intermittent preventive therapy for malaria in pregnancy at clearing infections and preventing low birth weight: A prospective, multi-country observational study across Africa, submitted). In a recent study in Mali and Burkina Faso, where the triple dhfr mutant was detected in $<50 \%$ and the dhps K540E mutation was detected in less than $1 \%$ of samples, the overall PCR uncorrected failure rate was $4.9 \%$ [33]. In Benin, where the dhfr/dhps quadruple mutant was detected in $>80 \%$ of parasite isolates, but dhps K540E was not detected, $11 \%$ of women with positive smear at the time of IPTp-SP administration had parasites detected by microscopy on follow-up within one month [34]. In Zambia, where the dhfr/dhps quintuple mutant was detected in parasites in $63 \%$ of women, failure rates were $26 \%$ by 35 days, [28] while in this site in Malawi, where parasites from $95 \%$ of women carried the quintuple mutant, failure rates were $32.5 \%$ at 28 days and $38.9 \%$ by day 35. While failure rates in these modified in vivo efficacy studies appear to correlate quite well with the prevalence of SP resistance mutations, there is poor correlation between SP resistance and IPTp-SP efficacy; the effect of IPTp-SP on infant birth weight or maternal haemoglobin remains remarkably constant over the spectrum of resistance, up to fixation of the quintuple mutant (Desai $M$, et al.: Impact of sulfadoxine-pyrimethamine resistance on effectiveness of intermittent preventive therapy for malaria in pregnancy at clearing infections and preventing low birth weight: A prospective, multi-country observational 
study across Africa, submitted). There are some data to suggest that the additional mutation at dhps A580G renders IPTp-SP ineffective, however, additional studies are needed to confirm this relationship. Given the expense and difficulties associated with conducting in vivo efficacy studies, and the correlation of failure rates with SP resistance mutations, consideration should be given to monitoring SP resistance markers instead of conducting in vivo efficacy studies. However, until a clearer relationship exists between the prevalence of molecular markers of SP resistance and the threshold at which IPTp-SP is no longer effective, IPTp-SP should continue to be delivered, ideally at each scheduled ANC visit, with close monitoring in areas where the sextuple mutant is emerging.

\section{Limitations}

Although this study suggests a beneficial effect of SP, the lack of a placebo arm impedes the ability to assess the true effect of SP treatment. It is possible that some women, particularly the multigravid ones, would have been able to clear the infections from their peripheral blood even without being treated with SP. However, until there is more evidence that IPTp-SP does not provide any benefit, it is not ethical to conduct such a study. Furthermore, as these women were not followed until delivery, it is not known whether the infections were cleared from the placenta, which remains a relatively protected site for malaria.

\section{Conclusion}

Despite fixation of the quintuple mutant in this population, SP retained some benefit when administered to asymptomatic parasitaemic pregnant women. The difference in parasite clearance in multigravid versus primigravid women highlights the importance of pre-existing immunity in clearing parasites. Although the prevalence of SP resistance has compromised the efficacy of SP as a treatment for acute malaria, it retains partial activity among pregnant women with asymptomatic parasitaemia, and is still useful when given as IPTp. Nonetheless, it remains important to continue to search for alternative regimens to prevent malaria in pregnancy.

\begin{abstract}
Abbreviations
ANC: Antenatal care; Cl: Confidence interval; dhfr: Dihydrofolate reductase; dhps: Dihydropteroate synthase; IPTp: Intermittent preventive treatment of malaria in pregnancy; IQR: Inter-quartile range; ITN: Insecticide-treated nets; LBW: Low birth weight; msp: Merozoite surface protein; PCR: Polymerase chain reaction; SP: Sulphadoxine-pyrimethamine; WHO: World Health Organization.
\end{abstract}

\section{Competing interests}

The authors declare that they have no competing interests.

\section{Authors' contributions}

JG participated in the analysis and drafted the manuscript. DM oversaw the data collection. REW performed the statistical analysis. JA assisted with the statistical analysis and performed the PCR assays. NCI performed the sequencing. YPS performed and interpereted molecular analyses. DPM participated in the design and coordination of the study. JS participated in the design and coordination of the study and helped to draft the manuscript. All authors read and approved the final manuscript.

\section{Acknowledgments}

The authors wish to thank Georgina Makuta, Orpha Malunga, Lameck Chimkango and all the staff at the ANC clinic of Machinga District Hospital, as well as the community members who participated in or otherwise contributed to these studies. This work was supported by the US President's Malaria Initiative, US Agency for International Development, under the terms of an Interagency Agreement with the Centers for Disease Control and Prevention (CDC) and through a Cooperative Agreement (Number 5 U01 Cl000189) between the CDC and the Malaria Alert Centre, College of Medicine. The sponsor of the study had no role in study design, data collection, data analysis, data interpretation, or writing of the report. The corresponding author had full access to all of the data in the study and had final responsibility for the decision to submit for publication.

\section{Disclaimer}

The findings and conclusions presented in this manuscript are those of the authors and do not necessarily reflect the official position of the US President's Malaria Initiative, US Agency for International Development, or US Centers for Disease Control and Prevention.

\section{Author details}

${ }^{1}$ Malaria Branch, Division of Parasitic Diseases \& Malaria, Centers for Disease Control and Prevention, Atlanta, GA, USA. ${ }^{2}$ Malaria Alert Centre, University of Malawi College of Medicine, Blantyre, Malawi. ${ }^{3}$ Rwanda Zambia HIV Research Group, Emory University, Atlanta, GA, USA. ${ }^{4}$ Department of Community Health, College of Medicine, Lilongwe, Malawi.

Received: 7 January 2015 Accepted: 23 April 2015

Published online: 13 May 2015

\section{References}

1. Desai M, ter Kuile FO, Nosten F, McGready R, Asamoa K, Brabin B, et al. Epidemiology and burden of malaria in pregnancy. Lancet Infect Dis. 2007;7:93-104

2. ter Kuile FO, van Eijk AM, Filler SJ. Effect of sulfadoxine-pyrimethamine resistance on the efficacy of intermittent preventive therapy for malaria control during pregnancy. JAMA. 2007;297:2603-16.

3. Kayentao K, Kodio M, Newman RD, Maiga H, Doumtabe D, Ongoiba A, et al. Comparison of intermittent preventive treatment with chemoprophylaxis for the prevention of malaria during pregnancy in Mali. J Infect Dis. 2005;191:109-16.

4. Parise ME, Ayisi JG, Nahlen BL, Schultz LJ, Roberts JM, Misore A, et al. Efficacy of sulfadoxine-pyrimethamine for prevention of placental malaria in an area of Kenya with a high prevalence of malaria and human immunodeficiency virus infection. Am J Trop Med Hyg. 1998;59:813-22.

5. WHO Malaria Policy Advisory Committee Secretariat. Malaria Policy Advisory Committee to the WHO: conclusions and recommendations of September 2012 meeting. Malar J. 2012;11:424.

6. Taylor SM, Antonia A, Chaluluka E, Mwapasa V, Feng G, Molyneux ME, et al. Antenatal receipt of sulfadoxine-pyrimethamine does not exacerbate pregnancy-associated malaria despite the expansion of drug-resistant Plasmodium falciparum: clinical outcomes from the QuEERPAM study. Clin Infect Dis. 2012;55:42-50.

7. Taylor SM, Antonia A, Feng G, Mwapasa V, Chaluluka E, Molyneux M, et al. Adaptive evolution and fixation of drug-resistant Plasmodium falciparum genotypes in pregnancy-associated malaria: 9-year results from the QuEERPAM study. Infect Genet Evol. 2012;12:282-90.

8. Kayentao K, Garner P, van Eijk AM, Naidoo I, Roper C, Mulokozi A, et al. Intermittent preventive therapy for malaria during pregnancy using 2 vs 3 or more doses of sulfadoxine-pyrimethamine and risk of low birth weight in africa: systematic review and meta-analysis. JAMA. 2013;309:594-604.

9. Feng G, Simpson JA, Chaluluka E, Molyneux ME, Rogerson SJ. Decreasing burden of malaria in pregnancy in Malawian women and its relationship to use of intermittent preventive therapy or bed nets. PLOS ONE. 2010;5:e12012.

10. Harrington WE, Mutabingwa TK, Muehlenbachs A, Sorensen B, Bolla MC, Fried $M$, et al. Competitive facilitation of drug-resistant Plasmodium 
falciparum malaria parasites in pregnant women who receive preventive treatment. Proc Natl Acad Sci U S A. 2009;106:9027-32.

11. Harrington $W$, Mutabingwa $T$, Kabyemela $E$, Fried M, Duffy $P$. Intermittent treatment to prevent pregnancy malaria does not confer benefit in an area of widespread drug resistance. Clin Infect Dis. 2011;53:224-30.

12. Minja D, Schmiegelow C, Mmbando B, Boström S, Oesterholt M, Magistrado P, et al. Infections with Plasmodium falciparum sextuple dihydrofolate reductase/ dihydropteroate synthetase allelic haplotypes during pregnancy are associated with decreased birth weight in Korogwe, Tanzania. Emerg Inf Dis. 2013,19 Sept http://dx.doi.org/10.3201/eid1909.130133

13. Gutman J, Kalilani L, Taylor S, Zhou Z, Wiegand RE, Thwai KL, et al. Plasmodium falciparum dhps-581G mutation reduces the effectiveness of sulfadoxine-pyrimethamine intermittent preventive therapy in Malawian pregnant women. JID 2015. Epub ahead of print.

14. Msyamboza K, Amanor A, Kazembe P, Brabin B, Meshnick S, Mwapasa V. In-vivo parasitological response to sulfadoxine-pyrimethamine in pregnant women in southern Malawi. Malawi Med J. 2007;19:11-3.

15. WHO. Standard protocol for measuring efficacy of antimalarial drugs in high transmission settings. Geneva: World Health Organization; 2003.

16. Iriemenam N, Shah M, Gatei W, van Eijk A, Ayisi J, Kariuki S, et al. Temporal trends of sulphadoxine-pyrimethamine (SP) drug-resistance molecular markers in Plasmodium falciparum parasites from pregnant women in western Kenya. Malar J. 2012;11:134.

17. Kublin JG, Dzinjalamala FK, Kamwendo DD, Malkin EM, Cortese JF, Martino LM, et al. Molecular markers for failure of sulfadoxine-pyrimethamine and chlorproguanil-dapsone treatment of Plasmodium falciparum malaria. J Infect Dis. 2002;185:380-8

18. WHO. Recommended Genotyping Procedures (RGPS) to identify parasite populations, Developed after an informal consultation organized by the Medicines for Malaria Venture and cosponsored by the World Health Organization, 29-31 May 2007, Amsterdam, The Netherlands. 2007. http://www.who.int/malaria/publications/atoz/rgptext_sti.pdf, accessed Dec 9, 2014.

19. Kaplan EL, Meier P. Nonparametric estimation from incomplete observations. J Amer Statist Assn. 1958;53:457-81.

20. Groenwold RH, Donders AR, Roes KC, Harrell Jr FE, Moons KG. Dealing with missing outcome data in randomized trials and observational studies. Am J Epidemiol. 2012;175:210-7.

21. Cox DR. Regression models and life tables. J Royal Stat Soc Ser B. 1972:20:187-220.

22. Schwartz G. Estimating the dimension of a model. Ann Stat. 1978;6:461-4.

23. Wickham H. ggplot2: elegant graphics for data analysis. New York: Springer; 2009.

24. WHO. Methods for surveillance of antimalarial drug efficacy. Geneva: World Health Organization; 2009.

25. WHO. Updated $\mathrm{WHO}$ policy recommendation: intermittent preventive treatment of malaria in pregnancy using sulfadoxine-pyrimethamine (IPTp-SP). http://www.who.int/entity/malaria/publications/atoz/ who_iptp_sp_policy_recommendation/en/index.htm

26. Gutman J, Mwandama D, Wiegand R, Ali D, Mathanga DP, Skarbinski J. Effectiveness of intermittent preventive treatment with sulfadoxinepyrimethamine in pregnancy on maternal and infant birth outcomes in Machinga District, Malawi. J Inf Dis. 2013;208:907-16.

27. Arinaitwe E, Ades V, Walakira A, Ninsiima B, Mugagga O, Patil TS, et al. Intermittent preventive therapy with sulfadoxine-pyrimethamine for malaria in pregnancy: a cross-sectional study from Tororo, Uganda. PLoS ONE. 2013:8:e73073.

28. Tan KR, Katalenich BL, Mace KE, Nambozi M, Taylor SM, Meshnick SR, et al. Efficacy of sulphadoxine-pyrimethamine for intermittent preventive treatment of malaria in pregnancy, Mansa, Zambia. Malar J. 2014;13:227.

29. Mace K, Chalwe V, Katalenich BL, Nambozi M, Mubikayi L, Mulele CK, et al. Evaluation of sulphadoxine-pyrimethamine for intermittent preventive treatment of malaria in pregnancy: a retrospective birth outcomes study-Mansa, Zambia. Malar J. 2015;14:69.

30. White N. Intermittent presumptive treatment for malaria. PLoS Med. 2005;2:e3.

31. Cohee L, Kalilani-Phiri L, Boudova S, Joshi S, Mukadam R, Seydel K, et al. Submicroscopic malaria infection during pregnancy and the impact of intermittent preventive treatment. Malar J. 2014;13:274.
32. Tagbor H, Bruce J, Agbo M, Greenwood B, Chandramohan D. Intermittent screening and treatment versus intermittent preventive treatment of malaria in pregnancy: a randomised controlled non-inferiority trial. PLOS ONE. 2010;5:e14425.

33. Coulibaly S, Kayentao K, Taylor S, Guirou E, Khairallah C, Guindo N, et al. Parasite clearance following treatment with sulphadoxine-pyrimethamine for intermittent preventive treatment in Burkina-Faso and Mali: 42-day in vivo follow-up study. Malar J. 2014;13:41.

34. Moussiliou A, De Tove YS-S, Doritchamou J, Luty A, Massougbodji A, Alifrangis $\mathrm{M}$, et al. High rates of parasite recrudescence following intermittent preventive treatment with sulphadoxine-pyrimethamine during pregnancy in Benin. Malar J. 2013;12:195.

\section{Submit your next manuscript to BioMed Central and take full advantage of:}

- Convenient online submission

- Thorough peer review

- No space constraints or color figure charges

- Immediate publication on acceptance

- Inclusion in PubMed, CAS, Scopus and Google Scholar

- Research which is freely available for redistribution 